\section{(2) OPEN ACCESS}

\title{
Non-vitamin K antagonist oral anticoagulants, proton pump inhibitors and gastrointestinal bleeds
}

\author{
Joris Komen (1) , ${ }^{1,2}$ Anton Pottegård, ${ }^{3}$ Paul Hjemdahl, ${ }^{4}$ Aukje K Mantel-Teeuwisse, ${ }^{1}$ \\ Björn Wettermark, ${ }^{5}$ Maja Hellfritzsch, ${ }^{3}$ J Hallas, ${ }^{3}$ Ron Herings, ${ }_{1}^{6}$ Lisa Smits, ${ }^{6}$ \\ Thomas Forslund, ${ }^{4}$ Olaf Klungel ${ }^{1}$
}

- Additional supplemental material is published online only. To view, please visit the journal online (http://dx.doi. org/10.1136/heartjnl-2021319332)

Clinical Pharmacology and Pharmacoepidemiology, Utrecht Institute of Pharmaceutical Sciences, Utrecht University, Utrecht, The Netherlands ${ }^{2}$ Department of Healthcare Development, Stockholm Region Public Healthcare Services Committee, Stockholm, Sweden ${ }^{3}$ Clinical Pharmacology and Pharmacy, Department of Public Health, University of Southern Denmark, Odense, Denmark ${ }^{4}$ Department of Medicine, Solna Karolinska Institute, Stockholm, Sweden

${ }^{5}$ Department of Pharmacy, Pharmacoepidemiology and Social Pharmacy, Uppsala University, Uppsala, Sweden ${ }^{2}$ PHARMO Institute, Utrecht, The Netherlands

Correspondence to

Joris Komen, Utrecht University, Utrecht 3508 TC, The Netherlands; j.j.komen@uu.nl

Received 11 March 2021 Accepted 2 July 2021

\section{ABSTRACT \\ Objective To evaluate if proton pump inhibitor (PPI) treatment reduces the risk of upper gastrointestinal bleeding (UGIB) in patients with atrial fibrillation (AF) treated with non-vitamin $\mathrm{K}$ antagonist oral anticoagulants (NOACs).}

Design We used a common protocol, common data model approach to conduct a cohort study including patients with AF initiated on a NOAC in Stockholm, Denmark and the Netherlands from April 2011 until July 2018. The outcome of interest was a UGIB diagnosed in a secondary care inpatient setting. We used an inverse probability weighted (IPW) Poisson regression to calculate incidence rate ratios (IRRs), contrasting PPI use to no PPI use periods.

Results In 164290 NOAC users with AF, providing 272570 years of follow-up and 39938 years of PP exposure, 806 patients suffered a UGIB. After IPW, PPI use was associated with lower UGIB rates (IRR: $0.75 ; 95 \% \mathrm{Cl}: 0.59$ to 0.95$)$. On an absolute scale, the protective effect was modest, and was found to be largest in high-risk patients, classified as age 75-84 years (number needed to treat for 1 year (NNTY): 787), age $\geq 85$ years (NNTY: 667), HAS-BLED score $\geq 3$ (NNTY: 378) or on concomitant antiplatelet therapy (NNTY: 373). Conclusion Concomitant treatment with a PPI in NOAC-treated patients with AF is associated with a reduced risk of severe UGIB. This indicates that PPI cotreatment can be considered, in particular among the elderly patients, patients with a HAS-BLED score $\geq 3$, and/or in patients on concomitant antiplatelet therapy.

\section{INTRODUCTION}

Pooled results from clinical trials showed that treatment with non-vitamin $\mathrm{K}$ antagonist oral anticoagulants (NOACs) significantly increased the risk of upper gastrointestinal bleeding (UGIB) compared with warfarin. ${ }^{1}$ Proton pump inhibitors (PPIs) reduce gastric acid production and prevent ulcer recurrence. ${ }^{2}$ In patients on aspirin treatment, which increases the risk of GIB, ${ }^{3}$ PPIs have been shown to reduce the risk of GIB. ${ }^{4}$ Therefore, PPI use is recommended for patients on aspirin treatment with certain comorbidities and comedications. ${ }^{5}$ Since clinical trials show an overall increased risk of UGIB associated with NOAC treatment, it is hypothesised that cotreatment with a PPI could decrease the risk of UGIB in NOAC users as well.
An observational study from the USA showed markedly reduced risks of UGIB associated with PPI use in patients treated with NOACs. ${ }^{6}$ In contrast, the COMPASS trial showed no protective effect with respect to GI bleeding overall, while a subanalysis on gastroduodenal bleeding showed a clearly reduced risk. ${ }^{7}$ However, this trial was in patients with stable cardiovascular disease and peripheral artery disease receiving a lower dose of rivaroxaban than in atrial fibrillation (AF) (5 mg two times per day).

In the absence of convincing results, the guidelines state that PPI treatment may be considered to reduce the risk of GIB, especially in those with a history of GI bleeding or ulcer and patients requiring concomitant use of (dual) antiplatelet therapy, ${ }^{8}$ a statement that was, however, removed in the most recent guidelines. ${ }^{9}$ As there is currently limited evidence from randomised studies regarding the effect of PPIs on UGIB in NOAC-treated patients with AF, observational data are the main source of guidance for this clinically relevant topic. Therefore, the aim of the current study was to assess the association between PPI use and UGIBs in patients with AF treated with a NOAC in three Western European countries.

\section{METHODS}

\section{Database}

For this population-based cohort study, we used three different databases: the Swedish Healthcare Database in the Stockholm region (complete population, $\mathrm{n}=2.3$ million), the nationwide Danish health registers (complete population, $\mathrm{n}=5.8$ million) and the PHARMO Database (random sample from the Dutch population, $\mathrm{n}=4$ million). The databases are described in detail elsewhere. ${ }^{10-12}$ All three databases contain prescription claims data from community pharmacies, registered by Anatomical Therapeutic Chemical codes, and all three databases contain medical diagnostic data from secondary care, registered by 10 th revision of the International Statistical Classification of Diseases and Related Health Problems (ICD-10) codes. In addition, the Stockholm database also contains medical diagnostic data from primary care, also registered by ICD-10 codes. We used a common protocol and a common data model to combine the data from the different databases. 


\section{Study population}

From each database, we included all patients dispensed a NOAC with a known history of AF, defined by a registration of the ICD-10 code I48 any time prior to or within 90 days after the first NOAC dispensing, to account for diagnostic lag. ${ }^{13}$ Patients entered the cohort at the date of their first ever NOAC prescription (cohort entry date), and we included patients from April 2011 until July 2018. We considered a patient to be on continued NOAC treatment when the patient claimed a prescription for a NOAC within 30 days after the calculated end of the previous prescription (see online supplemental figure 1). We censored patients at an outcome of interest, at the calculated end of their last prescription, when they died, moved out of the region or database, or switched to warfarin treatment. Patients could re-enter the cohort after they stopped their treatment if restarting NOAC therapy, and follow-up was defined in a similar manner after re-entering the cohort. All patients had to have at least 3 years of follow-up time prior to cohort entry, in order to adequately assess baseline characteristics.

\section{Exposure definition}

During follow-up, patients were considered to be exposed to PPIs when they claimed a PPI prescription (see online supplemental figure 1). They were considered exposed until the end of the duration of their last consecutive PPI prescription. We considered PPI treatment to be consecutive if a new prescription was claimed within the duration of the prior prescription, with another 30-day grace period added to account for irregular fill patterns and minor non-compliance. We calculated the duration of the prescription using the number of tablets dispensed, thus assuming a one tablet a day dosing regimen. To avoid bias from reverse causality (ie, that patients receive a PPI because of suspected or early symptoms of a UGIB), we used a lag time of 7 days after a first PPI prescription before we considered a patient exposed to PPI.

\section{Outcome definition}

The outcome of interest was a diagnosis code indicating a severe UGIB (see online supplemental table 1 for ICD-10 codes). We defined a severe UGIB as a registration of such a bleed in secondary inpatient care. Using this approach for the outcome, validation studies have shown a positive predictive value (PPV) of 98.1 and sensitivity of $82.3 \%$ for the Stockholm database, ${ }^{14}$ and a PPV of 98.0 and a sensitivity of $89.5 \%$ for the Danish database. $^{12}$

\section{Covariate assessment}

Given the non-random allocation of PPIs, potentially introducing confounding by indication, adjustment was needed. We adjusted for age, sex, year of inclusion, days from cohort entry date as well as relevant baseline comorbidities, time-varying comorbidities and time-varying comedications.

Baseline covariates included comorbidities in the HAS-BLED score (except labile international normalised ratio): hypertension, renal disease, liver disease, stroke history, prior bleeding or anaemia, and alcohol abuse; and comorbidities in the $\mathrm{CHA}_{2} \mathrm{DS}_{2}$ VASc score, not represented in the HAS-BLED score: heart failure, vascular disease and diabetes (see online supplemental table 1 for ICD-10 codes). We searched for registrations of relevant diagnosis codes in the 3 years prior to each patient's cohort entry date.

Time-varying comorbidities included: peptic ulcer, GI cancer, gastritis, oesophagitis, gastro-oesophageal reflux disease or dyspepsia, abdominal pain, lower GI problems and other GI problems (see online supplemental table 1 for ICD-10 codes). As these comorbidities might be markers for an already present UGIB, we added a 7-day lag period to the actual registration date of the diagnosis, to avoid reverse causality in the assessment of covariates. In addition, as these confounders might change over time, and affect both the risk of UGIB and the chance of PPI prescription, we partitioned follow-up time into 91-day periods, with the individual patient's initial cohort entry date as starting point. We searched for registrations of these diagnosis codes in the 3 years prior to the first day of the 91-day period. We defined the time-varying comorbidities as acute if the code was registered in the 30 days prior to the first day, as current if it was registered in the 30-90 days prior to the first day, as recent if it was registered in the 90-365 days prior to the first day, and as long-term if it was registered in the 365 days -3 years prior to the first day.

The comedications assessed were aspirin, non-steroidal antiinflammatory drugs (NSAIDs), clopidogrel, other antiplatelets, oral corticosteroids, diuretics, beta blockers, calcium channel blockers, renin-angiotensin-aldosterone system inhibitors, statins, oral antidiabetic drugs, insulins and antidepressants (see online supplemental table 1 for ATC codes). As comedications may change over time, we used the same 91-day periods as for the time-varying confounders. We looked for a prescription in the 180 days prior to the first day of the 91-day period.

\section{Statistical analysis}

We used descriptive statistics to present baseline characteristics for each database. To describe PPI users and non-users, we defined patients as users of a PPI if they received a PPI at some point during follow-up. This division was only done to describe the cohorts, as for all other analyses we used time-varying exposure definitions to define PPI exposed periods in order to avoid immortal time bias. ${ }^{15}$

Given the time-varying exposure and time-varying covariates, we used time-varying Poisson regression to calculate adjusted incidence rate ratios (IRRs) with 95\% CIs for the association between PPI use and UGIB (see online supplemental file 1 for the rationale for the statistical analysis). We used time-varying inverse probability weights (IPWs) to account for confounding introduced by the included covariates. We calculated 90-day period-specific probabilities of receiving PPI treatment conditional on the aforementioned covariates using a logistic regression model. The time-varying covariates were included as categorical variables, with the timing of the diagnoses considered, as described above. For each 91-day period, the IPW was calculated by dividing the prevalence of observed PPI treatment during follow-up by the probability of receiving treatment, to obtain a stabilised IPW. All statistical analyses were performed with statistical software R V.4.0.0 and RStudio Desktop V.1.1.463. We considered a $\mathrm{p}$ value of $<0.05$ as statistically significant.

\section{Meta-analysis}

The analyses could not be conducted centrally on a pooled database due to privacy regulations but were performed locally and separately in the three databases. All study centres used the same protocol, same programming code, and same ICD-10 codes for outcomes and comorbidity codes through a common data model. For all analyses, the results from each database were pooled using a meta-analysis. We performed a Cochran's Q statistic to test for heterogeneity across the databases and used a fixed effects meta-analysis based on the results from this test. 
Table 1 Summary of baseline characteristics per database

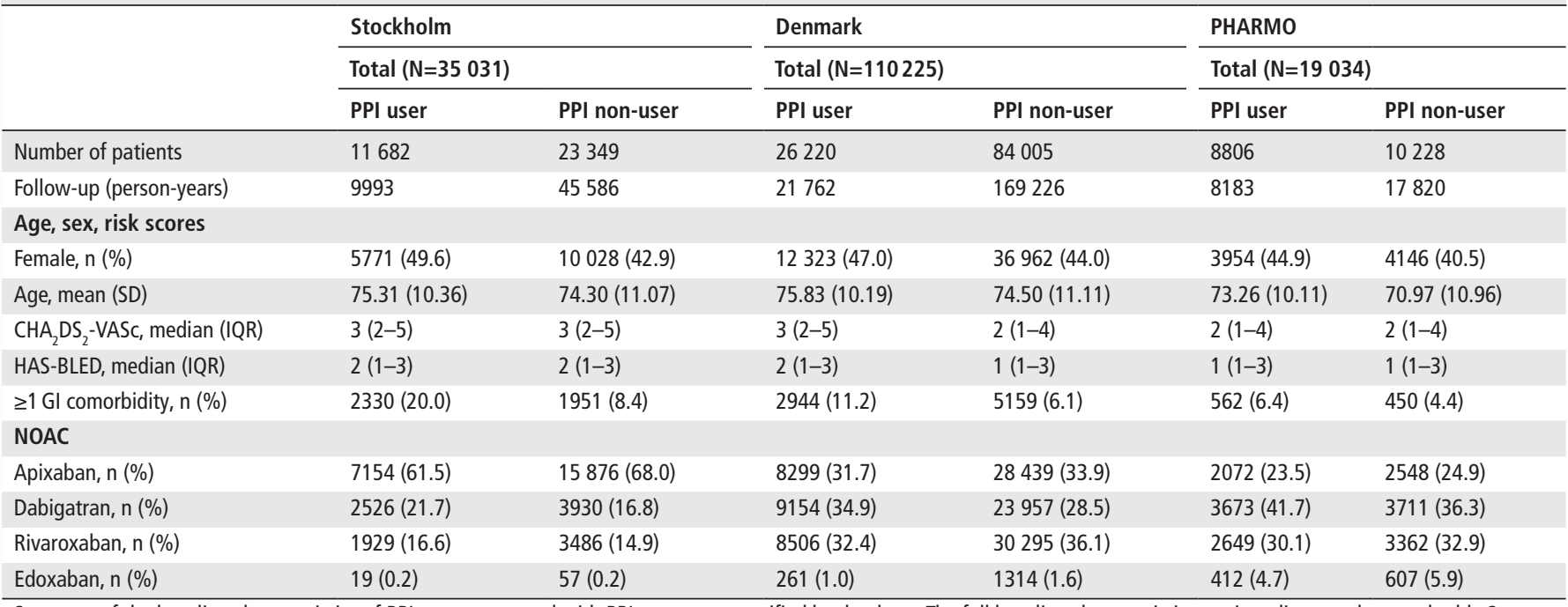

Summary of the baseline characteristics of PPI users compared with PPI non-users stratified by database. The full baseline characteristics are in online supplemental table 2 . $\mathrm{Gl}$, gastrointestinal; NOAC, non-vitamin K antagonist oral anticoagulant; PPI, proton pump inhibitor.

\section{Supplementary analyses}

In addition to the main analyses, we performed several stratified and sensitivity analyses. These analyses are described in the eMethods in the online supplemental file 1. In short, we stratified by sex, age, bleeding risk and individual NOAC. We performed six sensitivity analyses to test the robustness of our findings when varying our analytical choices.

\section{RESULTS}

\section{Cohort characteristics}

In total, we included 164290 NOAC users with AF in the study, of whom 46708 (28\%) used a PPI at some point during follow-up (see table 1 for a summary and online supplemental table 2 for all baseline characteristics). The mean age of the PPI users was slightly higher than for non-users, and women used PPIs more often in all three databases. In Stockholm, apixaban was the most frequently used NOAC (>60\%), while in Denmark and PHARMO all NOACs, except edoxaban, were used to approximately the same extent during the study period. Both the mean HAS-BLED and $\mathrm{CHA}_{2} \mathrm{DS}_{2}$-VASc scores were higher in PPI users compared with non-users. Patients receiving PPIs more often had GI comorbidities. In total, the cohorts accumulated 272570 person-years (pys) of NOAC use of which 39938 pys were exposed to PPIs. PPIs were most commonly used in the PHARMO Database with 31\% of all follow-up time being exposed to PPI, while this was $11 \%$ in Denmark and 18\% in Stockholm. In Stockholm, omeprazole was the most frequently used PPI (72\%), while in Denmark this was pantoprazole (60\%), and in the PHARMO both were used approximately equally (51\% pantoprazole and $41 \%$ omeprazole).

\section{Associations between PPI use and UGIB}

A total of 806 severe UGIBs occurred during 272570 pys of follow-up yielding an overall IR of $0.30 \% / p y$. The pooled unadjusted (crude) IRR for exposed versus non-exposed person-time was 1.06 (95\% CI: 0.86 to 1.30 ). The cohorts were however imbalanced regarding several baseline characteristics. After IPW, all covariates had a standardised mean difference below 0.1 , indicating successful weighting (online supplemental figure $2 \mathrm{~A}-\mathrm{C}$ ). Taking the time-varying IPW into account, the pooled IRR for UGIB was 0.75 (95\% CI: 0.59 to 0.95 ), indicating a protective effect of PPIs on UGIBs (see figure 1). The adjusted IRRs were consistent in all three databases: 0.79 (95\% CI: 0.49 to 1.26$)$ in Stockholm, $0.72(95 \%$ CI: 0.53 to 0.97 ) in Denmark and 0.85 (95\% CI: 0.39 to 1.85 ) in PHARMO.

\section{Stratified results}

The incidence of UGIB increased with increasing age groups, as did the protective effect of PPIs, which was greatest in patients above the age of 75 years (75-84 IPW IRR: 0.60 ; 95\% CI: 0.39 to 0.93 , $\geq 85$ IPW IRR: $0.64 ; 95 \%$ CI: 0.40 to 1.03$)$. The numbers needed to treat for 1 year (NNTYs) in these groups were 788 and 668, respectively. Patients with a HAS-BLED score of 3 or more experienced twice as many UGIBs as patients with a score below $3(0.52 \% /$ py vs $0.22 \% / p y)$, and the protective effect of PPIs was largest in this group as well (IPW IRR 0.51; 95\% CI: 0.35 to 0.77 , NNTY: 378 ). Patients with concomitant antiplatelet use had the highest crude rate of UGIB (0.64\%/py) and the protective effect of PPI treatment was significantly greater than in patients without concomitant antiplatelet use (IPW IRR: 0.64; $95 \%$ CI: 0.39 to 1.05 , NNTY: 374$)$. Stratifying by sex and concomitant NSAID use yielded no statistically different results.

The protective effect of PPIs on UGIB was only present in patients receiving apixaban or dabigatran (IPW IRR: $0.65 ; 95 \%$ CI: 0.43 to 0.98 and $0.65 ; 95 \%$ CI: 0.39 to 1.08 , respectively) but not in patients receiving rivaroxaban $(1.06 ; 95 \% \mathrm{CI}: 0.73$ to 1.54$)$ (see table 2).

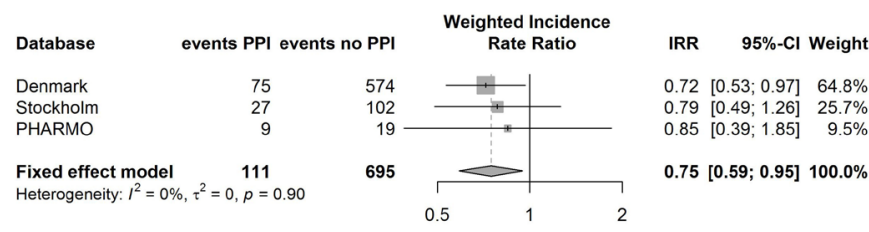

Figure 1 Results from the meta-analysis on the inverse probability weighted incidence rate ratio (IRR) of upper gastrointestinal bleeds. PPI, proton pump inhibitor. 
Table 2 Crude and adjusted incidence rate ratios (IRRs) of upper gastrointestinal bleeds per subgroup

\begin{tabular}{|c|c|c|c|c|c|c|}
\hline & $\mathrm{n}$ events & Follow-up time (person-years) & Incidence rate (\%/year) & Crude IRR (95\% Cl) & IPW IRR (Cl) & LRT significant* \\
\hline \multicolumn{6}{|l|}{ Age } & 2 out of 3 \\
\hline $0-64$ & 53 & 43542 & 0.12 & 2.55 (1.33 to 4.88$)$ & 1.09 (0.48 to 2.48$)$ & \\
\hline $65-74$ & 252 & 101012 & 0.25 & 1.50 (1.06 to 2.11$)$ & 0.99 (0.65 to 1.49$)$ & \\
\hline $75-84$ & 294 & 87954 & 0.33 & 0.71 (0.49 to 1.03 ) & 0.58 (0.37 to 0.89$)$ & \\
\hline$\geq 85$ & 207 & 40063 & 0.52 & 0.74 (0.49 to 1.12 ) & 0.67 (0.42 to 1.07$)$ & \\
\hline \multicolumn{6}{|l|}{ Sex } & 0 out of 3 \\
\hline Female & 457 & 149597 & 0.31 & 1.02 (0.77 to 1.34 ) & 0.66 (0.47 to 0.92$)$ & \\
\hline Male & 349 & 122974 & 0.28 & 1.12 (0.83 to 1.51$)$ & $0.88(0.62$ to 1.24$)$ & \\
\hline \multicolumn{6}{|l|}{ HAS-BLED } & 3 out of 3 \\
\hline Low (0-2) & 472 & 209553 & 0.23 & 1.14 (0.86 to 1.52$)$ & 0.95 (0.7 to 1.29$)$ & \\
\hline High $(\geq 3)$ & 334 & 63018 & 0.53 & 0.76 (0.57 to 1.03 ) & 0.54 (0.36 to 0.8$)$ & \\
\hline \multicolumn{6}{|c|}{ Concomitant NSAID } & 1 out of 3 \\
\hline No & 691 & 249520 & 0.28 & 1.01 (0.81 to 1.27$)$ & 0.74 (0.57 to 0.96$)$ & \\
\hline Yes & 115 & 23050 & 0.50 & 1.15 (0.72 to 1.86$)$ & 0.84 (0.46 to 1.54$)$ & \\
\hline \multicolumn{6}{|c|}{ Concomitant AP } & 2 out of 3 \\
\hline No & 585 & 239339 & 0.24 & $1.10(0.87$ to 1.4$)$ & 0.80 (0.61 to 1.06$)$ & \\
\hline Yes & 221 & 33232 & 0.67 & 0.79 (0.54 to 1.16$)$ & 0.63 (0.38 to 1.04$)$ & \\
\hline \multicolumn{6}{|l|}{ NOAC } & 2 out of 3 \\
\hline Apixaban & 282 & 93566 & 0.30 & 0.93 (0.67 to 1.31 ) & 0.67 (0.45 to 1.01$)$ & \\
\hline Dabigatran & 240 & 100105 & 0.24 & 1.06 (0.71 to 1.58 ) & 0.64 (0.39 to 1.07 ) & \\
\hline Rivaroxaban & 278 & 76842 & 0.36 & 1.29 (0.92 to 1.80$)$ & 1.03 (0.71 to 1.50$)$ & \\
\hline
\end{tabular}

Number of events, follow-up time, incidence rate, crude IRR and IPW IRR of PPI versus no PPI exposure in different subgroups.

*The number of databases in which the LRT was significant. If this test was significant in two or more databases, we considered a subgroup as a relevant effect modifier.

AP, antiplatelet; IPW, inverse probability weighted; LRT, likelihood ratio test; NOAC, non-vitamin K antagonist oral anticoagulant; NSAID, non-steroidal anti-inflammatory drug;

PPI, proton pump inhibitor.

\section{Sensitivity analysis}

The results from the sensitivity analyses are presented in the eResults section in the online supplemental file 1 . None of the sensitivity analyses showed different results compared with the main analysis.

\section{DISCUSSION}

In this large multicountry population-based study, covering 162 333 NOAC-treated patients with AF, we found that PPI use was associated with a $25 \%$ reduced risk of UGIB during NOAC treatment. This result was consistent in all three databases. The protective effect was most pronounced in high-risk patients, that is, patients above the age of 75 years, and patients with a HASBLED score of 3 or higher and/or on concomitant antiplatelet therapy. Interestingly, the protective effect of PPIs was only observed in those treated with apixaban or dabigatran and not in those treated with rivaroxaban.

Our results are in line with prior observational research and evidence from the only randomised controlled trial available. ${ }^{67}$ The COMPASS trial, comparing pantoprazole with placebo in patients treated with rivaroxaban $5 \mathrm{mg}$ two times per day, reported an HR of 0.93 (95\% CI: 0.60 to 1.47 ) for all upper GI events, while for a UGIB confirmed by endoscopy or radiography, the HR was 0.25 (95\% CI: 0.07 to 0.89 ). ${ }^{7}$ However, these results were from patients with stable cardiovascular disease instead of patients with $\mathrm{AF}$, and using a lower dose of rivaroxaban than recommended in $\mathrm{AF}$ ( $5 \mathrm{mg}$ two times per day instead of $15-20 \mathrm{mg}$ once daily). A recent large observational study from the USA reported an adjusted IRR of 0.66 (95\% CI: 0.52 to 0.85 ) for UGIB in OACtreated patients with AF using PPIs, however, this study also included patients on warfarin therapy. ${ }^{6}$ In line with our findings, this study also reported the largest risk reduction in patients receiving apixaban and dabigatran (adjusted IRRs of 0.50 and 0.51 , respectively), but contrary to our findings, they also found a protective effect in patients receiving rivaroxaban, although lower (IRR 0.68). Potential explanations could be that rivaroxaban is taken only once daily resulting in higher peak plasma concentrations or that rivaroxaban should always be taken with food, both of which could influence PPI effectiveness. However, given the wide CIs in our NOAC subgroups, we believe these differences between the NOACs could also be a play of chance.

\section{Clinical implications}

As there is currently no randomised trial assessing the efficacy of PPIs in NOAC-treated patients with AF, and our results are in line with the COMPASS trial and another large observational study, ${ }^{67}$ we believe that PPI cotreatment can be considered for the prevention of UGIBs in high-risk NOAC-treated patients with AF (age above 75 years, a HAS-BLED score above 2, and/or receiving concomitant antiplatelet therapy). The NNTYs were 788 (age 75-84 years), 668 (age $\geq 85$ years), 378 (HAS-BLED >2) and 374 (antiplatelet). Given that NOAC treatment is lifelong, more realistic NNTYs might be for a 5 -year period, which would yield NNTYs of 158, 134, 76 and 75, respectively. In addition, we used a conservative endpoint by only including specific ICD-10 codes in hospitalised patients. Therefore, the absolute risk of UGIB in our study was low (approximately three times lower than in the clinical trials ${ }^{1}$ ) and with that, the absolute risk reduction could potentially be higher if the absolute risks were as high as in the clinical trials. It is also conceivable that our results are to some extent affected by residual confounding, since PPIs were primarily channelled to high-risk patients. However, this implies that the true effect is most likely larger than we could demonstrate.

\section{Limitations}

Our study has several limitations. First, despite using timevarying IPW, there is still the potential for residual confounding, 
for example, due to lifestyle factors such as smoking. Second, there was potential misclassification of exposure, as we used prescription claims data, which have potentially biased the point estimate towards a neutral association. ${ }^{16}$ In addition, in all three settings, PPIs can be bought over-the-counter. Therefore, we might have classified some patients as non-users, while in reality they were using over-the-counter PPIs. Third, we used a conservative approach to define the outcome of interest and might therefore underestimate the true number of events. On the other hand, a conservative approach leads to a higher PPV for the outcome and a lower risk of detection bias. Fourth, NOACs can also be prescribed for other indications than AF, and we have not included patients with those diagnoses. Fifth, as we lacked data on renal function, we were not able to assess the appropriateness of NOAC dosing.

\section{Strengths}

Our study has several strengths. First, we used data from three different European countries; two unselected populations and the PHARMO database which is a random sample, yielding generalisable results to similar populations, also supported by the consistent results in all databases. Second, our results were robust to all sensitivity analyses, indicating that our study results are independent of the analytical choices we made. Third, this is the first study addressing this clinically important question in a European healthcare setting, where prescribing patterns are probably different than in a US setting. In addition, there are currently no randomised trial data addressing the clinical question of the efficacy of PPI in NOAC users and thus observational research can provide guidance.

\section{CONCLUSION}

We found an association between PPI use and a lower risk of severe UGIB in an unselected NOAC-treated population with $\mathrm{AF}$, which was consistent in three different Western European healthcare settings. Based on these findings, as well as the results of other studies, we believe PPIs can be useful to reduce the risk of UGIBs in NOAC-treated patients with AF with a high risk of bleeds.

\section{Key messages}

What is already known on this subject?

- Non-vitamin K antagonist oral anticoagulant (NOAC) use in patients with atrial fibrillation increases the risk of gastrointestinal bleeds.

- Treatment with proton pump inhibitors (PPIs) can reduce the risk of gastrointestinal bleeds.

\section{What might this study add?}

- In patients with atrial fibrillation from Stockholm, Denmark and the Netherlands receiving NOACs, $28 \%$ received a PPI somewhere during follow-up.

- PPI use was associated with a $25 \%$ risk reduction in hospitalisation for upper gastrointestinal bleeds.

- This protective effect was largest in patients above age 75 years, in patients with a high bleeding risk and in patients receiving concomitant antiplatelet therapy.

\section{How might this impact on clinical practice?}

- Especially in high-risk patients, PPI therapy may be considered in patients with atrial fibrillation treated with NOACs.
Acknowledgements We would like to thank Morten Olesen from the University of Southern Denmark and Josine Kuipers from the PHARMO Institute for their help with the data management.

Contributors $\mathrm{JK}, \mathrm{AP}, \mathrm{PH}, \mathrm{MH}, \mathrm{JH}, \mathrm{TF}$ and $\mathrm{OK}$ were involved in designing the study. $\mathrm{JK}, \mathrm{AP}$ and $\mathrm{RH}$ were involved in analysing the data. All authors were involved in drafting and critically revising the manuscript. All authors approved the final version of the manuscript.

Funding The authors have not declared a specific grant for this research from any funding agency in the public, commercial or not-for-profit sectors.

Competing interests JK reports personal fees from Boehringer Ingelheim, outside the submitted work. AP reports grants from Alcon, grants from Almirall, grants from Astellas, grants from AstraZeneca, grants from Boehringer Ingelheim, grants from Novo Nordisk, grants from Servier, grants from LEO Pharma, outside the submitted work. JH reports grants from AstraZeneca, outside the submitted work. RH and LS report other fees from University of Utrecht, during the conduct of the study.

\section{Patient consent for publication Not required.}

Ethics approval The study was approved by the Regional Ethical Review Board in Stockholm (EPN 2015/579-31/2). Informed consent was not required in this registry study of anonymised data.

Provenance and peer review Not commissioned; externally peer reviewed.

Data availability statement Data may be obtained from a third party and are not publicly available. Ethical and privacy reasons prohibit sharing of data from all three databases.

Supplemental material This content has been supplied by the author(s). It has not been vetted by BMJ Publishing Group Limited (BMJ) and may not have been peer-reviewed. Any opinions or recommendations discussed are solely those of the author(s) and are not endorsed by BMJ. BMJ disclaims all liability and responsibility arising from any reliance placed on the content. Where the content includes any translated material, BMJ does not warrant the accuracy and reliability of the translations (including but not limited to local regulations, clinical guidelines, terminology, drug names and drug dosages), and is not responsible for any error and/or omissions arising from translation and adaptation or otherwise.

Open access This is an open access article distributed in accordance with the Creative Commons Attribution Non Commercial (CC BY-NC 4.0) license, which permits others to distribute, remix, adapt, build upon this work non-commercially, and license their derivative works on different terms, provided the original work is properly cited, appropriate credit is given, any changes made indicated, and the use is non-commercial. See: http://creativecommons.org/licenses/by-nc/4.0/.

\section{ORCID iD}

Joris Komen http://orcid.org/0000-0002-5471-3132

\section{REFERENCES}

1 Ruff CT, Giugliano RP, Braunwald E, et al. Comparison of the efficacy and safety of new oral anticoagulants with warfarin in patients with atrial fibrillation: a metaanalysis of randomised trials. Lancet 2014:383:955-62.

2 Brunner G, Creutzfeldt W. Omeprazole in the long-term management of patients with acid-related diseases resistant to ranitidine. Scand I Gastroentero/ Suppl 1989;166:101-5. discussion 111-3.

3 Derry S, Loke YK. Risk of gastrointestinal haemorrhage with long term use of aspirin: meta-analysis. BMJ 2000;321:1183-7.

4 Yeomans N, Lanas A, Labenz J, et al. Efficacy of esomeprazole (20 mg once daily) for reducing the risk of gastroduodenal ulcers associated with continuous use of lowdose aspirin. Am J Gastroenterol 2008;103:2465-73.

5 Hamm CW, Bassand J-P, Agewall S, et al. ESC guidelines for the management of acute coronary syndromes in patients presenting without persistent ST-segment elevation: the task force for the management of acute coronary syndromes (ACS) in patients presenting without persistent ST-segment elevation of the European Society of cardiology (ESC). Eur Heart J 2011;32:2999-3054.

6 Ray WA, Chung CP, Murray KT, et al. Association of oral anticoagulants and proton pump inhibitor Cotherapy with hospitalization for upper gastrointestinal tract bleeding. JAMA 2018;320:2221.

7 Moayyedi P, Eikelboom JW, Bosch J, et al. Pantoprazole to prevent gastroduodenal events in patients receiving rivaroxaban and/or aspirin in a randomized, double-blind, placebo-controlled trial. Gastroenterology 2019;157:403-12.

8 Steffel J, Verhamme P, Potpara TS, et al. The 2018 European Heart Rhythm Association practical guide on the use of non-vitamin $\mathrm{K}$ antagonist oral anticoagulants in patients with atrial fibrillation. Eur Heart J 2018;39:1330-93.

9 Arbelo E, Bax JJ. 2020 ESC guidelines for the diagnosis and management of atrial fibrillation developed in collaboration with the European association of CardioThoracic surgery (EACTS) ESC Committee for practice guidelines (CpG) and national cardiac societies document reviewers, and Author/Task force member affiliations: listed in the appendix 


\section{Arrhythmias and sudden death}

10 Forslund T, Wettermark B, Wändell P, et al. Risk scoring and thromboprophylactic treatment of patients with atrial fibrillation with and without access to primary healthcare data: experience from the Stockholm health care system. Int J Cardiol 2013;170:208-14.

11 PHARMO. Institute for drug outcomes research. Available: www. pharmo.nl [Accessed 23 Jan 2020].

12 Schmidt M, Schmidt SAJ, Sandegaard JL, et al. The Danish national patient registry: a review of content, data quality, and research potential. Clin Epidemiol 2015:7:449-90.

13 Hellfritzsch M, Pottegård A, Haastrup SB, et al. Cohort selection in register-based studies of direct oral anticoagulant users with atrial fibrillation: an inevitable trade-off between selection bias and misclassification. Basic Clin Pharmacol Toxicol 2020;127:bcpt.13423.

14 Friberg L, Skeppholm M. Usefulness of health registers for detection of bleeding events in outcome studies. Thromb Haemost 2016;116:1131-9.

15 Suissa S. Immortal time bias in pharmaco-epidemiology. Am J Epidemiol 2008;167:492-9.

16 Jurek AM, Greenland S, Maldonado G, et al. Proper interpretation of nondifferential misclassification effects: expectations vs observations. Int J Epidemiol 2005;34:680-7 